\title{
ESTUDIOS
}

\section{Las agencias estadounidenses de evaluación de la Responsabilidad Social Empresarial'}

\section{Marta Camprodon Rosanas, José Sols Lucia y Albert Florensa Giménez $^{2}$}

Palabras clave: Inversión Socialmente Responsable (ISR), Social Investment Forum (SIF), Inversión ética, Responsabilidad Social Corporativa (RSC), Agencias de evaluación de la RSC, Responsabilidad Social de la Empresa (RSE), ética empresarial, stakeholders.

Key words: Socially Responsible Investment (SRI), Social Investment Forum (SIF), Ethical Investment, Corporate Social Responsibility (CSR),Agencies for the evaluation of CSR, responsible business, business ethics, stakeholders.

Mots clés : Investissement Socialement Responsable (ISR), investissement éthique, Responsabilité Sociale Corporative (RSC), Agences d'évaluation de la RSC, Responsabilité Sociale de l'entreprise (RSE), éthique patronale, stakeholders.

\footnotetext{
' La investigación para la realización de este estudio ha sido subvencionada por la Cátedra Ethos Ramon Llull (Universidad Ramon Llull, Barcelona); este ha exigido la realización de numerosas entrevistas a responsables de las agencias estadounidenses aquí estudiadas. Esta tarea fue llevada a cabo por Marta Camprodon durante el mes de octubre del 2007. Esta fuente propia no será citada cada vez que se utilice.

${ }^{2}$ Los tres autores son investigadores, profesores en la Facultad de Economía IQS (Instituto Químico de Sarrià, Universidad Ramon Llull, Barcelona), miembros de la Cátedra de Ética y Pensamiento Cristiano del IQS, así como del Laboratorio de Análisis y Crítica Social (LACS) del mismo centro.
} 


\section{Introducción}

En nuestro artículo "Las agencias de evaluación de la Responsabilidad Social Corporativa: estudio de un caso", publicado en esta misma revista, analizamos las agencias europeas de evaluación de la Responsabilidad Social Corporativa $\left(\mathrm{RSC}^{3}\right.$. Aquí continuaremos aquella investigación analizando las agencias de los Estados Unidos. Para realizar esta investigación, en octubre del 2007, se realizaron distintas entrevistas a tres agencias de evaluación de la RSC de EEUU. Las agencias fueron Innovest, Calvert y KLD.

Conviene que recordemos, en primer lugar, algunas ideas-clave que expusimos en aquel artículo. Es sabido que la inquietud por conocer a dónde iba el dinero depositado en los bancos y por buscar que su inversión fuera coherente con los ideales propios de los inversores nació en el siglo XVIII, en Estados Unidos, con los cuáqueros, aunque fue en el siglo pasado cuando se extendió esta inquietud en la sociedad norteamericana, y posteriormente también en Europa.

Durante las últimas décadas, muchas empresas han querido ser socialmente responsables y aparecer públicamente como tales. Quieren mostrarse ante la sociedad como empresas competentes financieramente y preocupadas por la globalización y la falta de normativas globales, el respeto del medio ambiente, de los derechos humanos, de las condiciones laborales de sus trabajadores, del trato dado a sus clientes y a sus proveedores; en resumen, muchas empresas del siglo XXI están preocupadas por la Responsabilidad Social Corporativa (RSC), o también denominada Responsabilidad Social de la Empresa (RSE).

Debido a estos dos grandes factores que acabamos de explicar (inquietud ética de algunos inversores y preocupación de algunas empresas por la RSC) nacen las agencias de evaluación de la RSC. Estas agencias analizan el grado de RSC de las empresas y normalmente las comparan con el sector al que pertenecen para ver si son o no innovadoras en relación con la RSC en su sector. Hacen tres tipos de análisis distintos ofreciendo así tres servicios diferentes: la evaluación declarativa, la evaluación solicitada y los índices de responsabilidad social. No todas las agencias ofrecen los tres servicios.

La evaluación declarativa consiste en el análisis del grado de responsabilidad social de la empresa basándose en informaciones públicas. Normalmente se analizan

${ }^{3}$ Cfr. M. CAMPROdOn - J. Sols - A. Florensa (2006), 393-422. Sobre esta misma temática: M. CAMPROdON - J. Sols. - A. Florensa (2007); F. Déjean (2005), 21-23; y D. Dupré, I. Girerd-Potin (2002), 91-94. 
empresas que cotizan en bolsa. Para realizar este estudio, la agencia de evaluación no necesita el consentimiento de la empresa analizada, ya que ella no es la destinataria del informe a realizar, y, tal y como hemos dicho, éste se elabora a partir de informaciones públicas. La agencia evalúa la relación de la empresa con los distintos "stakeholders" y hace un análisis comparativo con empresas del mismo sector. A partir de la información pública disponible, cada agencia elabora su propia base de datos. Los informes realizados por la agencia son vendidos a inversores institucionales o a gestores de fondos, y posteriormente son utilizados para crear carteras de valores. El cuadro 1, "Fuentes de información para analizar la RSC", recoge las fuentes de información que utilizan cinco agencias de distintos países para realizar esta evaluación declarativa de la RSC: Vigeo de Francia, Eiris del Reino Unido, SAM de Suiza, y KLD e Innovest de Estados Unidos. A pesar de ser agencias de distintos países, puede verse claramente en este cuadro cómo existe una base común en las fuentes de información. Estas cinco agencias de evaluación utilizan documentos públicos para realizar el análisis declarativo de la empresa y toda la información que recogen la utilizan para crear sus propias bases de datos.

\section{CUADRO I. Fuentes de información para analizar la RSC}

\begin{tabular}{|c|c|c|c|c|c|}
\hline & Vigeo & Eiris & Innovest & SAM & KLD \\
\hline $\begin{array}{l}\text { Documentos } \\
\text { públicos }\end{array}$ & 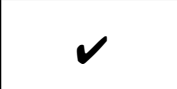 & $\checkmark$ & $v$ & $\checkmark$ & $\checkmark$ \\
\hline $\begin{array}{l}\text { Creación de } \\
\text { bases de datos }\end{array}$ & $\checkmark$ & $\checkmark$ & $\checkmark$ & $\checkmark$ & $\checkmark$ \\
\hline $\begin{array}{l}\text { Cuestionario a } \\
\text { empresas }\end{array}$ & $x$ & $\checkmark$ & $\checkmark$ & $\checkmark$ & $\checkmark$ \\
\hline $\begin{array}{l}\text { Entrevista a } \\
\text { empresas }\end{array}$ & $\checkmark$ & Ocasional & $\checkmark$ & Ocasional & Ocasional \\
\hline $\begin{array}{l}\text { Consulta de } \\
\text { páginas web }\end{array}$ & Ocasional & $x$ & Ocasional & Ocasional & $x$ \\
\hline $\begin{array}{l}\text { Cuestionario a } \\
\text { stakeholders }\end{array}$ & Ocasional & $x$ & $x$ & Ocasional & $\checkmark$ \\
\hline $\begin{array}{l}\text { Entrevista a } \\
\text { stakeholders }\end{array}$ & $x$ & Ocasional & Ocasional & Ocasional & Ocasional \\
\hline
\end{tabular}

Fuente: E. Alberola y S. Giamporcaro-Saunière (2006) 
Por su parte, la evaluación solicitada consiste en el análisis del grado de responsabilidad social de una empresa por requerimiento explícito de ésta. En esta evaluación se puede analizar tanto un solo departamento como toda la empresa. En ambos casos, se compara el departamento o la empresa con otras empresas del sector para estudiar en qué puntos la empresa es pionera en RSC, para a continuación potenciarlos, y en qué aspectos la empresa debería mejorar. La agencia evaluadora nunca podrá vender estos informes a terceros (sean quienes sean: inversores institucionales, gestores de fondos, empresas, periodistas, investigadores, políticos...), ya que el informe pertenece exclusivamente a la empresa analizada, y sólo ella tiene derecho a publicarlo.

Finalmente, otro producto que también pueden ofrecer las agencias de evaluación de la RSC son los índices de responsabilidad social. Estos índices están formados por las empresas mejor puntuadas en la evaluación declarativa. Normalmente, están constituidos por empresas que cotizan en bolsa. Habitualmente, una agencia de evaluación de la RSC analiza todas las empresas que pertenecen a un índice bursátil. Las empresas que obtengan mejor puntuación aparecerán en un índice de responsabilidad social elaborado por la agencia evaluadora. No obstante, debemos recordar que cada agencia de evaluación utiliza una metodología propia. Así pues, una empresa podría aparecer en el índice de responsabilidad social de una agencia, por haber sido bien evaluada por ella, y, en cambio, obtener mala puntuación en el estudio elaborado por otra agencia. Hay agencias que no tienen ningún índice de responsabilidad social, y hay agencias que tienen más de uno. Por ejemplo, Eiris ha creado los índices FTSE4Good, basándose en los índices bursátiles FTSE; Vigeo ha creado el índice Aspi Euro Zona a partir del Dow Jones Eurostoxx 600; y SAM Group ha creado los índices Dow Jones Sustainability a partir de diversos índices bursátiles Dow Jones.

A continuación, en el apartado 2, definiremos qué entendemos por Inversión Socialmente Responsable (ISR) y presentaremos una clasificación. Después compararemos la ISR de EEUU con la europea. En el apartado 3 explicaremos las agencias de evaluación de la RSC estadounidenses. Las clasificaremos y analizaremos tres de ellas en más profundidad. Finalmente, en el apartado 4 , haremos una comparación sintética entre las agencias de evaluación estadounidenses con las europeas. 


\section{La Inversión Socialmente Responsable en Estados Unidos y en Europa}

\section{I. Definición de ISR}

Estados Unidos fue el precursor de la Inversión Socialmente Responsable y continúa siendo el país que más la promueve. Antes de centrarnos en las ISR norteamericana $^{4}$ y europea ${ }^{5}$, nos gustaría analizar el concepto de ISR, ya que éste varía de un continente a otro, e incluso de un país a otro. Al conocer lo que cada país entiende por ISR y ver las diferencias semánticas, se comprenden mejor las discrepancias existentes en los análisis de la RSC de las empresas que llevan a cabo las agencias de evaluación. En el cuadro 2, "Descripción y definición de la ISR por los distintos SIFs europeos y norteamericanos", se recogen las definiciones que nos ofrecen los "Social Investment Forums" (SIF) de distintos países. El SIF es un foro que promueve la ISR. Existe a nivel continental, como es el caso del EUROSIF, pero también a nivel nacional, como serían los SIF de Estados Unidos, del Reino Unido o de Francia. En España no hay ningún SIF. Analizando las definiciones expuestas, se perciben matices distintos que cada país da a la ISR. Así pues, la definición americana presenta un enfoque más centrado en los valores, mientras que las definiciones europeas se orientan más a aspectos concretos, ya sean sociales, financieros o medioambientales. En Europa, el énfasis puesto en los tres aspectos mencionados está influido por el concepto de desarrollo sostenible, que tiene mayor fuerza en Europa que en los Estados Unidos. La definición americana no tiene en cuenta los aspectos financieros de la ISR. Cada vez se utiliza menos el término "inversión ética" porque suena a excesivamente religioso y moral para algunos oídos. Según Louche y Lydenberg (2006), en Estados Unidos la ISR se basa en los siguientes tres pilares: 1 / preselección de cartera o filtros de selección, 2 / inversiones en la comunidad y 3 / activismo de los accionistas. En cambio, en Europa los tres principales criterios son definidos como: 1 / preselección de cartera o filtros de selección, 2/ compromiso (diálogo con las empresas), y $3 /$ activismo accionarial y ejercicio del derecho de voto. En Europa, la inversión comunitaria no se considera ISR; en Estados Unidos, el activismo accionarial engloba el ejercicio del derecho de voto.

${ }^{4}$ Cfr. L. Albareda - M.R. Balaguer - D. Arenas (2007), 41-51; Social Investment Forum (2006); C. LOUCHE - S. LYDENBERG (2006)

${ }^{5}$ Cfr. L. Albareda - M.R. Balaguer - D. Arenas (2007), 31-40; C. Louche - S. Lydenberg (2006); M.R. Balaguer (2006), 78-84; Avanzi SRI Research (2005), Avanzi SRI Research (2006), Avanzi SRI Research - Vigeo Italla (2007). 


\section{CUADRO 2. Descripción y definición de la ISR por distintos SIF de Europa y de Estados Unidos}

\begin{tabular}{|c|c|c|}
\hline $\begin{array}{c}\text { Social } \\
\text { Investment } \\
\text { Forum (SIF) } \\
\end{array}$ & $\begin{array}{l}\text { Concepto } \\
\text { utilizado }\end{array}$ & Definición \\
\hline $\begin{array}{l}\text { Estados } \\
\text { Unidos }\end{array}$ & $\begin{array}{l}\text { Inversión } \\
\text { socialmente } \\
\text { responsable }\end{array}$ & $\begin{array}{l}\text { Integrar los valores personales y las preocupaciones } \\
\text { sociales en la inversión. }\end{array}$ \\
\hline $\begin{array}{l}\text { EUROSIF } \\
\text { (SIF de } \\
\text { Europa) }\end{array}$ & $\begin{array}{l}\text { Inversión } \\
\text { socialmente } \\
\text { responsable }\end{array}$ & $\begin{array}{l}\text { Asociar los objetivos financieros de los inversores } \\
\text { a las preocupaciones de éstos acerca de cuestiones } \\
\text { sociales, medioambientales y éticas. La ISR tiene en } \\
\text { cuenta tanto las necesidades financieras del inversor } \\
\text { como el impacto de la inversión en la sociedad. }\end{array}$ \\
\hline $\begin{array}{l}\text { Reino } \\
\text { Unido }\end{array}$ & $\begin{array}{l}\text { Inversión } \\
\text { socialmente } \\
\text { responsable }\end{array}$ & $\begin{array}{l}\text { Asociar los objetivos financieros de los inversores } \\
\text { a las preocupaciones de éstos acerca de cuestiones } \\
\text { sociales, medioambientales y éticas. }\end{array}$ \\
\hline Bélgica & $\begin{array}{l}\text { Inversión } \\
\text { sostenible y } \\
\text { socialmente } \\
\text { responsable }\end{array}$ & $\begin{array}{l}\text { Invertirde manera sostenible y socialmente responsable } \\
\text { significa: llevar a cabo una política que, tanto en su } \\
\text { formulación teórica como en su realización práctica, } \\
\text { tenga en cuenta, con el mismo grado de conciencia, } \\
\text { los efectos económicos, sociales, ecológicosy culturales } \\
\text { del proceso de inversión, tanto a corto como a largo } \\
\text { plazo; y significa también: dialogar con los grupos } \\
\text { sociales de interés en cuestión acerca de este tema. }\end{array}$ \\
\hline Francia & $\begin{array}{l}\text { Inversión } \\
\text { socialmente } \\
\text { responsable }\end{array}$ & $\begin{array}{l}\text { Reconocer que toda actividad económica produce } \\
\text { efectos, acerca de los cuales el inversor tiene una } \\
\text { parte de responsabilidad; decidir asumir esta res- } \\
\text { ponsabilidad, identificando la inversión realizada y } \\
\text { los riesgos adquiridos, y solidarizándose con la em- } \\
\text { presa beneficiaria; alargar su horizonte de inversión } \\
\text { al tiempo necesario para el desarrollo de todos los } \\
\text { efectos sociales, societales y medioambientales de la } \\
\text { actividad inducida por la inversión realizada. }\end{array}$ \\
\hline Alemania & $\begin{array}{l}\text { Inversión } \\
\text { sostenible }\end{array}$ & $\begin{array}{l}\text { Tomar en consideración, no sólo los aspectos financieros, } \\
\text { sino también los aspectos sociales, éticos y ecológicos. }\end{array}$ \\
\hline
\end{tabular}




\begin{tabular}{|l|l|l|}
\hline Italia & $\begin{array}{l}\text { Inversión } \\
\text { socialmente } \\
\text { responsable }\end{array}$ & $\begin{array}{l}\text { Tomar en consideración las cuestiones sociales y } \\
\text { éticas en el proceso de selección de inversiones y en } \\
\text { la gestión de éstas. }\end{array}$ \\
\hline Holanda & $\begin{array}{l}\text { Inversión } \\
\text { sostenible y y } \\
\text { ética }\end{array}$ & $\begin{array}{l}\text { Evaluar las inversiones posibles, no sólo en virtud de } \\
\text { criterios financieros, sino también en virtud de criterios } \\
\text { medioambientales y sociales. Este tipo de criterios } \\
\text { puede tener un carácter positivo (prueba de la visión } \\
\text { de futuro de la empresa) o un carácter negativo (lo } \\
\text { que revela aspectos inaceptables). }\end{array}$ \\
\hline Suecia & $\begin{array}{l}\text { Inversión } \\
\text { sostenible }\end{array}$ & $\begin{array}{l}\text { Una inversión que, además de criterios financieros, } \\
\text { incluya factores sociales, ecológicos y éticos en el } \\
\text { proceso de toma de decisión. }\end{array}$ \\
\hline
\end{tabular}

Fuente: LOUCHE-LYDENBERG (2006)

\subsection{El mercado de la ISR y sus estrategias}

Sánchez et al (2004) muestran cómo se divide el mercado de la ISR: "El mercado de la ISR se puede dividir en inversión minorista (el denominado mercado retail) e institucional. El primero está compuesto básicamente por los ahorros e inversiones particulares, mientras que el segundo está integrado por todo lo demás, especialmente los depósitos de planes de pensiones, aseguradoras y bancos. Así como en una primera fase fue el mercado minorista el que mostró mayor interés y ayudó a crear el mercado de la ISR, parece que en estos momentos la inversión institucional se erige como un elemento capaz de consolidar e incrementar la penetración de la ISR en los mercados financieros". ${ }^{7}$ Es necesario especificar que el mercado institucional es más voluminoso que el minorista.

La ISR comprende tres estrategias distintas, ${ }^{8}$ que vemos resumidas en el esquema 1 , "Estrategias de ISR". Veamos ahora con detalles en qué consisten estas estrategias.

\footnotetext{
"En España, siguiendo la recomendación de diferentes lingüistas, se traduce el adjetivo inglés "societal" y el francés "sociétal" por "social", sin distinguirlo de los adjetivos inglés y francés "social". Aquí, al ir los dos adjetivos juntos, excepcionalmente reproducimos el neologismo "societal", que nosotros no utilizamos.

7 P. Sánchez - M.A. Rodríguez - J.E. Ricart - S. Capdeville - E. Gal (2004), 14.

${ }^{8}$ Cfr. Kawamura (2002), 16-17 y Balaguer (2006), 79-84.
} 
ESQUEMA I.Estrategias de ISR

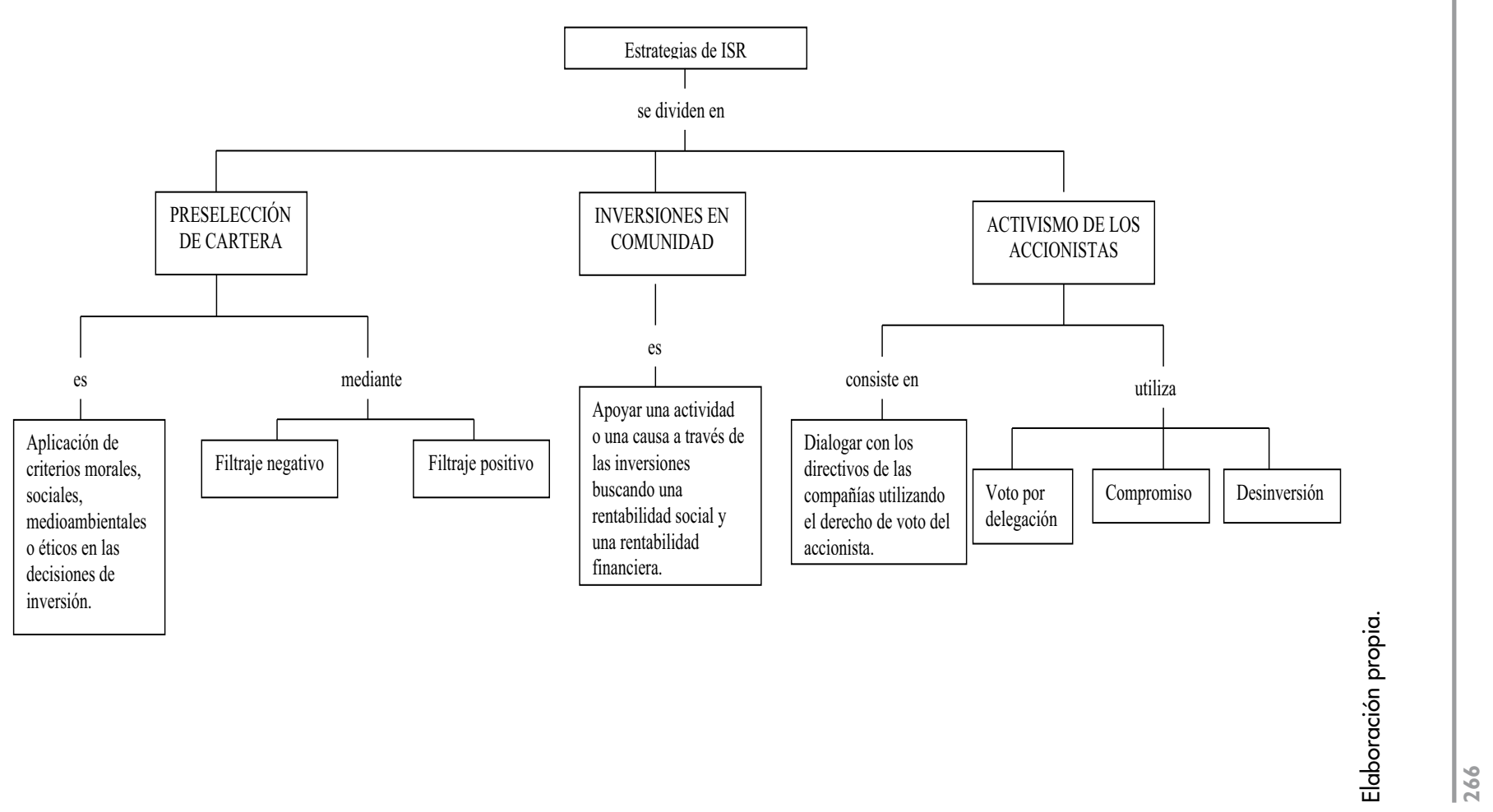


a) Preselección de la cartera o filtros de selección ("Screening"). La preselección de la cartera es una estrategia pasiva que consiste en aplicar criterios medioambientales, morales, éticos o sociales en las decisiones de inversión. Este filtraje de la ISR puede ser negativo o positivo.

En el filtro negativo, el inversor evita poner su dinero en empresas cuyas prácticas o cuyos productos vayan en contra de sus valores morales. En el cuadro 3 puede observarse los criterios de selección negativos más utilizados en Europa y en Estados Unidos. Ambos coinciden en excluir de sus inversiones cualquier empresa relacionada con el tabaco, el juego o el armamento. En el cuadro 4 puede verse un ejemplo de "checklist" de criterios negativos que KLD, agencia americana de evaluación de la RSC, pasa a sus clientes para conocer sus preferencias de inversión. Con este tipo de "checklist" la agencia de evaluación puede conocer el nivel de rechazo de un criterio. Por ejemplo, en la Sección 1 del cuadro 4, encontramos el criterio "alcohol". El inversor puede matizar en qué casos no está dispuesto en invertir en una empresa que fabrica alcohol: si la empresa obtiene más del $5 \%$ de los ingresos en la fabricación del alcohol, o si obtiene más del 10\%, o si obtiene más del $15 \%$.

Por su parte, en el filtro positivo, el inversor busca potenciar las empresas que hagan contribuciones positivas a la sociedad.

\section{CUADRO 3. Los criterios de selección mediante filtro negativo más utilizados en Europa y en Estados Unidos}

\begin{tabular}{|l|l|}
\hline \multicolumn{1}{|c|}{ Europa } & \multicolumn{1}{c|}{ Estados Unidos } \\
\hline Tabaco & Tabaco \\
\hline Juego & Juego \\
\hline Armamento & Armamento \\
\hline Energía nuclear & Alcohol \\
\hline $\begin{array}{l}\text { Violaciones de los derechos humanos, } \\
\text { trabajo infantil y regímenes totalitarios }\end{array}$ & Control de nacimientos/abortos \\
\hline Pornografía & No respeto al medioambiente \\
\hline $\begin{array}{l}\text { Maltrato a los animales y comercio de } \\
\text { pieles }\end{array}$ & $\begin{array}{l}\text { No respeto a la igualdad de oportuni- } \\
\text { dades en el trabajo }\end{array}$ \\
\hline
\end{tabular}

Fuente: LOUCHE-LYDENBERG (2006) 


\section{CUADRO 4. Ejemplo de "checklist" de criterios de selección mediante filtro negativo de la agencia KLD}

\section{Sección 1: Restricciones en negocios polémicos o controvertidos}

Armas de fuego. Esta restricción incluye empresas que:

- fabrican armas de fuego.

obtienen el $15 \%$ o más de los ingresos de la venta de armas de fuego.

- son propietarias de una empresa de armas de fuego.

son propiedad de una empresa de armas de fuego.

Alcohol. Esta restricción incluye empresas que:

- fabrican bebidas alcohólicas:

$\square$ que obtienen con ello más del $5 \%$ de los ingresos.

$\square$ que obtienen con ello más del $10 \%$ de los ingresos.

$\square$ que obtienen con ello más del $15 \%$ de los ingresos.

obtienen el $15 \%$ o más de los ingresos en la fabricación de productos necesarios para la producción de bebidas alcohólicas.

obtienen el $15 \%$ o más de los ingresos de la venta de alcohol.

- son propietarias de una empresa de alcohol.

- son propiedad de una empresa de alcohol

\section{Sección 2: Restricciones en cuestiones sociales}

Diversidad. Esta restricción incluye empresas que:

- han pagado importantes multas o sanciones civiles por haber estado implicadas en controversias o polémicas relacionadas con pleitos o juicios de la Comisión de Igualdad de Oportunidades de Empleo (Equal Employment Opportunities Commission).

Derechos humanos. Esta restricción incluye empresas que:

- tienen operaciones o invierten en Birmania, o utilizan este país como fuente de abastecimiento.

no respetan la soberanía, tierra, cultura, derechos humanos o propiedad intelectual de los pueblos indígenas, tanto de Estados Unidos como de otros países.

- tienen operaciones fuera de los Estados Unidos que han sido objeto, recientemente, de grandes polémicas en materia de derechos humanos, aunque aún no están registradas en clasificaciones KLD.

- desarrollan operaciones, fuera de los Estados Unidos, que recientemente han comportado importantes polémicas relativas a relaciones profesionales o a mano de obra; o bien desarrollan operaciones, dentro de los Estados Unidos, relacionadas con talleres clandestinos o con trabajo infantil. 
Sección 3: Restricciones de los clientes

Islámico: restricciones con el cerdo. Esta restricción incluye empresas que:

crían cerdos para su matanza o que producen carne de cerdo.

- matan cerdos o elaboran comida de cerdo.

- sirven carne de cerdo preparada como una parte importante de la actividad empresarial (obtienen más del 15\% de los ingresos de la venta de carne de cerdo).

Investigación con células madre embrionarias o con tejido fetal. Esta restricción incluye empresas que:

dirigen investigaciones con células madre embrionarias o con tejido fetal.

- proporcionan tecnologías para la investigación con células madre embrionarias o con tejido fetal.

u usan células fetales para el desarrollo de vacunas.

Fuente: KLD RESEARCH \& ANALYTICS 9

b) Inversiones en la comunidad ("community investing"). Esta estrategia consiste en apoyar una actividad o una causa a través de inversiones que buscan una rentabilidad social y una rentabilidad financiera. Ejemplos de estas inversiones podrían ser la financiación a cultivos biológicos, el desarrollo local, el desarrollo de la cultura, la atención a ancianos y a niños, o la atención a discapacitados. En Europa, este tipo de inversiones se relaciona e identifica, a veces, como "banca ética", por lo que, en general, las inversiones en comunidad no se consideran en la ISR. Alsina define un "banco ético" como aquel que "intenta conseguir simultáneamente dos objetivos: 1 / financiar actividades económicas que tengan un impacto social positivo, y 2/ obtener beneficios. Un banco ético sitúa estos dos objetivos al mismo nivel y se define justamente por el hecho de considerarlos, no sólo compatibles, sino necesariamente complementarios. Para ser un banco ético, obtener beneficios es indispensable, pero no suficiente; también es necesario que los beneficios se generen respetando una serie de criterios éticos básicos y financiando actividades con un impacto social positivo". ${ }^{10}$

Las inversiones en comunidad se parecen a la preselección de cartera con filtro positivo, ya que ambas potencian acciones o comportamientos positivos según

${ }^{9}$ Documento interno de KLD proporcionado por la propia empresa en la entrevista del 15 de octubre de 2007.

10 O. Alsina (2002), 27. 
criterios morales, éticos, sociales o medioambientales. La diferencia radica en que los filtros de selección con criterios positivos sirven para tomar decisiones de inversión sobre instrumentos financieros convencionales, como son las acciones y los fondos de inversión, mientras que las inversiones en comunidad normalmente apoyan proyectos.

c) Activismo de los accionistas ("shareholder advocacy"). Consiste en dialogar directamente con los directivos de las compañías utilizando el derecho básico del accionista, que es participar como copropietario en la toma de decisiones de la empresa a través del derecho a voto. El problema reside en que la mayoría de empresas tienen muchos accionistas, con lo que los pequeños accionistas raramente ejercen sus derechos. Con el activismo de los accionistas se pretende que tanto los propietarios de acciones como las organizaciones pro RSC unan sus derechos y hagan propuestas de comportamiento de la empresa acorde con sus ideales. Lo importante no es tanto que las propuestas reciban un apoyo de votos suficientes, sino que atraigan la atención de la dirección y del resto de accionistas. El activismo de los accionistas se concreta en tres vías:

c. 1) Voto por delegación ("Proxy voting"): Hacer propuestas de comportamiento de la empresa según los principios de RSC para que sean votadas en las asambleas de accionistas.

c.2) Compromiso ("Engagement"): Dialogar con los directivos de la empresa para impulsar políticas de RSC.

c.3) Desinversión ("Divestment"): Informar públicamente acerca de la venta de acciones que hacen algunos inversores por razones de desacuerdo con las políticas de empresa.

El Social Investment Forum crea una lista con los fondos de inversión éticos de Estados Unidos y los marca según preselección de cartera (si invierte en alcohol, tabaco, juego, defensa y armamento, pruebas con animales, medioambiente, derechos humano, relaciones laborales e igualdad de oportunidades en el empleo), según si hacen inversiones en la comunidad o no, y según si hacen activismo de los accionistas (voto por delegación) o no. En el Cuadro 5 puede verse una clasificación de los primeros 20 fondos de inversión éticos de la lista del Social Investment Forum. 
CUADRO 5. Preselección de cartera y activismo de los accionistas (por orden alfabético)

\begin{tabular}{|c|c|c|c|c|c|c|c|c|c|c|c|}
\hline $\begin{array}{l}\text { Nombre del Fondo de } \\
\text { Inversión }\end{array}$ & $\frac{\overline{0}}{\frac{0}{0}}$ & \begin{tabular}{l}
8 \\
8 \\
\hdashline
\end{tabular} & $\stackrel{\circ}{\stackrel{\$}{\Xi}}$ & 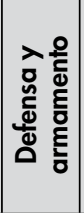 & 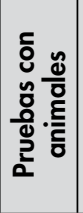 & 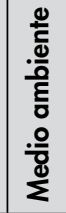 & 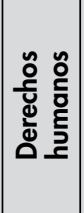 & 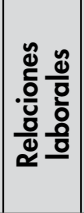 & 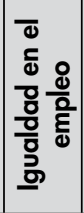 & 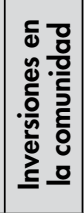 & 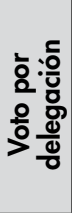 \\
\hline $\begin{array}{l}\text { Access Capital Strategies } \\
\text { Community Investment Fund }\end{array}$ & $x$ & $x$ & $x$ & $\mathrm{x}$ & $\mathrm{x}$ & NS & NS & NS & NS & $P$ & $\mathrm{X}$ \\
\hline Appleseed Fund & $\mathrm{R}$ & $\mathrm{R}$ & $\mathrm{R}$ & $\mathrm{R}$ & NS & $\mathrm{P}$ & $\mathrm{P}$ & $P$ & NS & $\mathrm{P}$ & $\mathrm{P}$ \\
\hline $\begin{array}{l}\text { Bridgeway Aggressive Investors } \\
2 \text { Fund }\end{array}$ & NS & $\mathrm{X}$ & NS & NS & NS & NS & NS & NS & NS & NS & $x$ \\
\hline Bridgeway Balanced Fund & NS & $x$ & NS & NS & NS & NS & NS & NS & NS & NS & $x$ \\
\hline $\begin{array}{l}\text { Bridgeway Blue Chip } 35 \text { Index } \\
\text { Fund }\end{array}$ & NS & $\mathrm{X}$ & NS & NS & NS & NS & NS & NS & NS & NS & $\mathrm{X}$ \\
\hline Bridgeway Large Cap Growth & NS & $X$ & NS & NS & NS & NS & NS & NS & NS & NS & $x$ \\
\hline Bridgeway Large Cap Value & NS & $\mathrm{X}$ & NS & NS & NS & NS & NS & NS & NS & NS & $x$ \\
\hline Bridgeway Small Cap Growth & NS & $x$ & NS & NS & NS & NS & NS & NS & NS & NS & $x$ \\
\hline Bridgeway Small Cap Value & NS & $x$ & NS & NS & NS & NS & NS & NS & NS & NS & $x$ \\
\hline $\begin{array}{l}\text { Bridgeway Ultra Small } \\
\text { Company Market Fund }\end{array}$ & NS & $\mathrm{X}$ & NS & NS & NS & NS & NS & NS & NS & NS & $x$ \\
\hline $\begin{array}{l}\text { Calvert Aggressive Allocation } \\
\text { Fund }\end{array}$ & $x$ & $\mathrm{X}$ & $\mathrm{x}$ & $\mathrm{R}$ & $\mathrm{R}$ & $P$ & $P$ & $P$ & $P$ & $P$ & $P$ \\
\hline Calvert Capital Accumulation A & $x$ & $x$ & $x$ & $\pi$ & $\mathrm{R}$ & $\mathrm{P}$ & $\mathrm{P}$ & $\mathrm{P}$ & $r$ & 1 & $\mathrm{P}$ \\
\hline Calvert Capital Accumulation B & $x$ & $x$ & $x$ & $\mathrm{R}$ & $\mathrm{R}$ & $\mathrm{P}$ & $\mathrm{P}$ & $\mathrm{P}$ & $\mathrm{P}$ & $\mathrm{P}$ & $\mathrm{P}$ \\
\hline Calvert Capital Accumulation C & $x$ & $x$ & $x$ & $\mathrm{R}$ & $\mathrm{R}$ & $\mathrm{P}$ & $\mathrm{P}$ & $P$ & $\mathrm{P}$ & $\mathrm{P}$ & $\mathrm{P}$ \\
\hline $\begin{array}{l}\text { Calvert Conservative Allocation } \\
\text { Fund }\end{array}$ & $x$ & $x$ & $\mathrm{x}$ & $\mathrm{R}$ & $\mathrm{R}$ & $P$ & $P$ & $P$ & $P$ & $P$ & $P$ \\
\hline $\begin{array}{l}\text { Calvert Global Alternative } \\
\text { Energy Fund A }\end{array}$ & NS & NS & NS & NS & NS & $P$ & $P$ & $P$ & $P$ & NS & $P$ \\
\hline $\begin{array}{l}\text { Calvert International } \\
\text { Opportunities Fund }\end{array}$ & $\mathrm{R}$ & $\mathrm{R}$ & NS & NS & NS & $P$ & $P$ & $P$ & $P$ & NS & $P$ \\
\hline Calvert Large Cap Growth A & $x$ & $x$ & $x$ & $R$ & $\mathrm{R}$ & $\mathrm{P}$ & $\mathrm{P}$ & $P$ & $\mathrm{P}$ & $\mathrm{P}$ & $\mathrm{P}$ \\
\hline Calvert Large Cap Growth B & $x$ & $x$ & $x$ & $R$ & $R$ & $\mathrm{P}$ & $\mathrm{P}$ & $\mathrm{P}$ & $\mathrm{P}$ & $\mathrm{P}$ & $\mathrm{P}$ \\
\hline Calvert Large Cap Growth C & $x$ & $X$ & $x$ & $\mathrm{R}$ & $\mathrm{R}$ & $\mathrm{P}$ & $\mathrm{P}$ & $P$ & $P$ & $\mathrm{P}$ & $\mathrm{P}$ \\
\hline
\end{tabular}

Leyenda: X: no inversión; P: inversión positiva; R: inversión restrictiva; NS: no filtro de selección.

Fuente: Social Investment Forum <http://www.socialinvest.org/resources/mfpc/screening.cfm> 


\subsection{Comparación de la ISR de Estados Unidos con Europa}

En la Figura 1 puede verse la ISR total de Estados Unidos, en el mercado minorista, entre los años 1995 y 2005, desglosada también según las tres estrategias anteriormente explicadas. En esta figura percibimos que la estrategia dominante es la preselección de cartera o filtros de selección ("screening"). También puede observarse que desde 1995 hasta 2005 se ha dado un incremento significativo en las inversiones socialmente responsables.

\section{FIGURA I. La Inversión Socialmente Responsable en \\ Estados Unidos (1995-2005)}

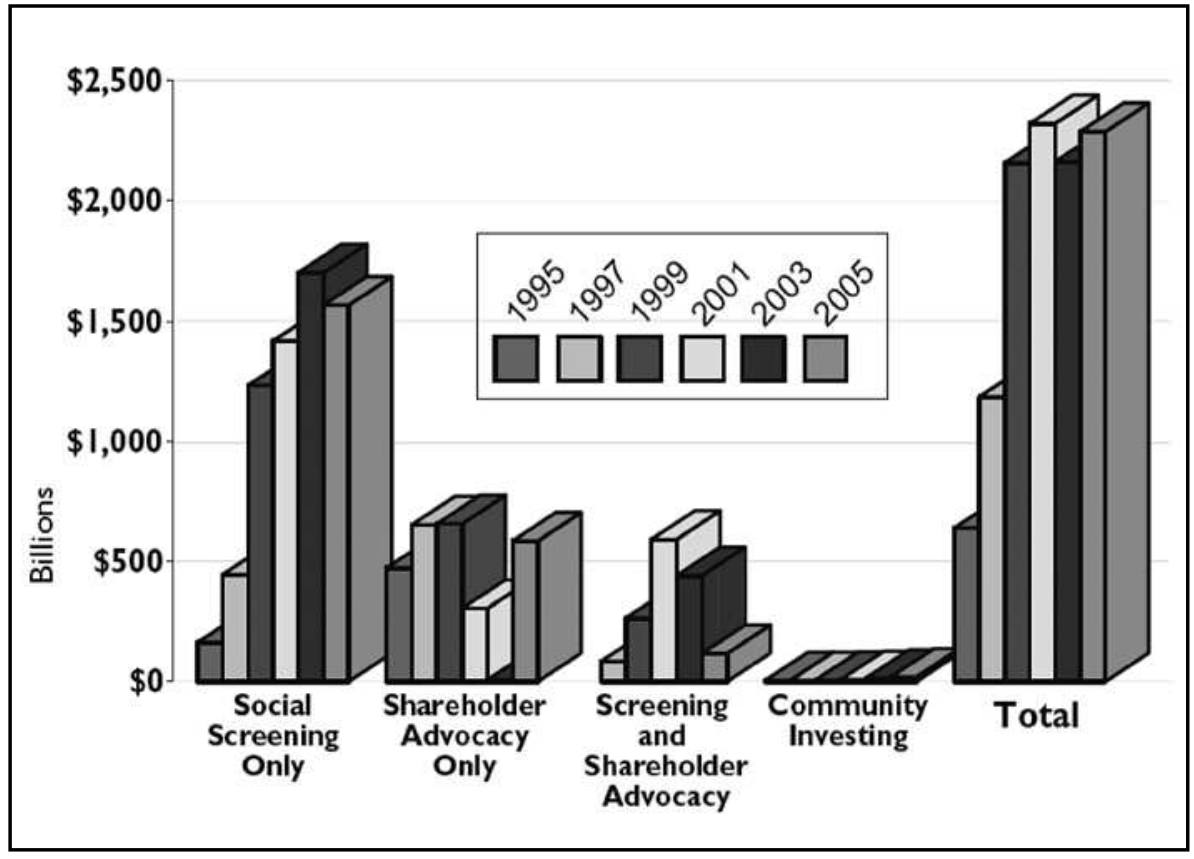

Unidad: miles de millones de \$ (= billones americanos)

Fuente: SOCIAL INVESTMENT FORUM (2006)

Sólo podemos comparar la ISR europea con la ISR norteamericana en su totalidad, ya que no hemos logrado dar con los datos desglosados en las distintas estrategias 
de la ISR europea. La diferencia entre la ISR en Estados Unidos y en Europa es abismal, tal y como se ve en el cuadro 6 "ISR en Europa y en Estados Unidos". Nos hemos permitido dos licencias: $1 /$ hacer la comparación entre dólares y euros, en lugar de hacerlo con una misma moneda, y $2 /$ no tener en cuenta la diferencia de paridad del poder adquisitivo en los EEUU y en Europa; y nos hemos otorgado esta doble licencia porque la diferencia entre la inversión norteamericana y la europea resulta, en cualquier caso, abismal. Eso sí, debemos decir, a modo de matiz, que la ISR europea sólo incluye los fondos de inversión UCITS."

\section{CUADRO 6. ISR en la Europa de los I5 y en Estados Unidos}

\begin{tabular}{|c|c|c|}
\hline Año & EEUU (en millones de \$) & Europa (en millones de $€$ ) \\
\hline 2003 & 2.164 .000 & 12.150 \\
\hline 2005 & 2.290 .000 & 24.127 \\
\hline 2007 & 2.702 .220 & 48.735 \\
\hline
\end{tabular}

Fuente: Avanzi SRI Research - Vigeo (2007), Social Investment Forum (2006) y First Affirmative Financial Network.

A pesar de la gran diferencia entre la ISR en Estados Unidos y la europea, lo que más nos interesa ahora es el porcentaje que cada una representa en la inversión total. En la Figura 2 se refleja el porcentaje que representa la ISR en la inversión total en la Europa de los 15 y en Estados Unidos. Salta a la vista que la ISR estadounidense es muy superior a la europea, al ser ésta de menos de un $1 \%$ y aquélla de un $12 \%$. Además, la ISR de Estados Unidos se incrementó un $2 \%$ del 2005 al 2006 , mientras que la europea se mantuvo estable, en torno al $0,6 \%$, durante ese mismo período.

$"$ UCITS son las siglas inglesas de "Undertakings for Collective Investments in Transferable Securities". Se refiere a la directiva de la Unión Europea que establece las condiciones bajo las cuales un fondo domiciliado en un país miembro de la Unión puede ser distribuido en los demás países miembros. El objetivo es simplificar las reglamentaciones en materia de inversiones y aumentar la protección del inversor. Si, por ejemplo, un fondo de inversión español está registrado en España, pero no puede ser comercializado en Europa, no estaría englobado en los fondos UCITS, por lo que no ha sido contabilizado en la Figura 2. 
FIGURA 2. Porcentaje de capital invertido en fondos socialmente responsables respecto al total de UCITS en la Europa de los 15 y en Estados Unidos

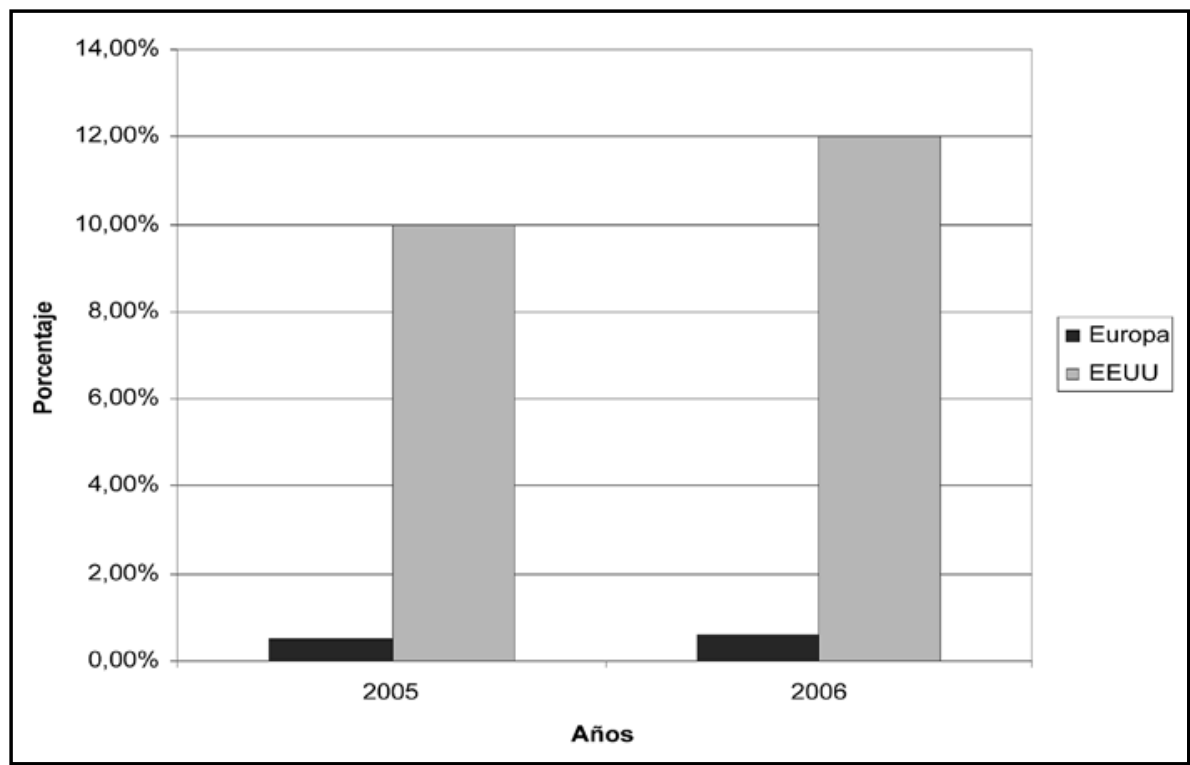

Fuente para los datos de Europa: AvanzI SRI RESEARCH (2006);

Fuente para los datos de Estados Unidos: SIF (2006) y NovETHIC (2007).

\section{Las agencias de evaluación norteamericanas y su clasificación}

Como señalábamos en la introducción a este estudio, las agencias de evaluación de la RSC pueden ofrecer tres servicios: la evaluación declarativa, la evaluación solicitada y los índices de la responsabilidad social. No obstante, no todas las agencias ofrecen los tres productos. 


\section{CUADRO 7. Clasificación de la evaluación que ofrecen las agencias de evaluación de la RSC}

\begin{tabular}{|c|c|c|c|c|}
\hline Agencias & $\begin{array}{c}\text { Sólo } \\
\text { evaluación } \\
\text { solicitada }\end{array}$ & $\begin{array}{c}\text { Sólo } \\
\text { evaluación } \\
\text { declarativa }\end{array}$ & $\begin{array}{l}\text { Evaluación } \\
\text { solicitada y } \\
\text { declarativa }\end{array}$ & $\begin{array}{l}\text { Tiene índice de } \\
\text { responsabilidad } \\
\text { social }\end{array}$ \\
\hline Europeas & $\begin{array}{l}\text { - BMJ } \\
\text { Ratings }\end{array}$ & $\begin{array}{l}\text { - Centre Info } \\
\text { - E-Capital } \\
\text { Partners } \\
\text { - Eiris } \\
\text { - Ethibel } \\
\text { - Inrate AG } \\
\text { - Scoris } \\
\text { - Siri } \\
\text { Company }\end{array}$ & $\begin{array}{l}\text { - AIS } \\
\text { - Avanzi } \\
\text { - CFIE } \\
\text { - Covalence } \\
\text { - Deminor Rating } \\
\text { - Dutch } \\
\text { Sustainability } \\
\text { Research BV } \\
\text { - Ethical Screening } \\
\text { - GES Investment } \\
\text { Services } \\
\text { - IMUG } \\
\text { - Oekom Research } \\
\text { - SAM Group } \\
\text { - SERM } \\
\text { - Trucost } \\
\text { - Vigeo } \\
\text { - EthiFinance }\end{array}$ & $\begin{array}{l}\text { - Avanzi } \\
\text { - GES } \\
\text { Investment } \\
\text { Services } \\
\text { - SAM Group } \\
\text { - Oekom } \\
\text { Research } \\
\text { - Vigeo } \\
\text { - E-Capital } \\
\text { Partners } \\
\text { - Eiris }{ }^{12} \\
\text { - Ethibel } \\
\text { - Siri Company }\end{array}$ \\
\hline Estadounidenses & & $\begin{array}{l}\text { - KLD } \\
\text { research } \\
\text { - Calvert } \\
\text { - Domini } \\
\text { Social } \\
\text { Investments }^{14} \\
\text { - Trillium } \\
\end{array}$ & - Innovest ${ }^{16}$ & $\begin{array}{l}\text { - Calvert } \\
\text { - Domini Social } \\
\text { Investments } \\
\text { - KLD research }\end{array}$ \\
\hline
\end{tabular}

Fuente: propia a partir de datos de ORSE - ADEME (2006).

${ }^{12}$ Eiris es una agencia de evaluación de la RSC europea con sede en Boston.

${ }^{13}$ Los informes de la evaluación solicitada que hace Calvert son de uso interno de esta agencia y no están a la venta.

${ }^{14}$ Los informes de la evaluación solicitada que hace Domini Social Investment son de uso interno de esta agencia y no están a la venta.

${ }^{15}$ Los informes de la evaluación solicitada que hace Trillium son de uso interno de esta agencia y no están a la venta.

${ }^{16}$ Innovest es una agencia de evaluación de la RSC americana con sede en París y Londres. 
En el Cuadro 7 "Clasificación de la evaluación que ofrecen las agencias de evaluación de la RSC" pueden verse las agencias de evaluación de la RSC estadounidenses y europeas clasificadas según los productos que ofrecen. Al observar este cuadro, remarcamos que sólo existe una agencia que se dedique exclusivamente a la evaluación solicitada: BMJ Ratings. En general, las agencias han optado o bien por realizar únicamente la evaluación declarativa, o bien por hacer los dos tipos de evaluación, pero sólo BMJ Ratings ofrece exclusivamente la solicitada. Esto es debido a que las agencias empezaron realizando la evaluación declarativa, después de lo cual, a medida que fueron creciendo, se dieron cuenta de que existía un nuevo mercado potencial en la evaluación solicitada. Algunas se lanzaron a él, pero sin dejar la evaluación con la que se habían estrenado, la declarativa; en definitiva, decidieron compatibilizar la evaluación pública de las empresas, haciendo informes para los inversores institucionales, con la evaluación privada, encaminada a conseguir que las empresas mejorasen sus niveles de responsabilidad social.

No obstante, no todas las agencias han seguido este camino. Por ejemplo, Eiris, que nació en Londres en 1983, decidió desde sus inicios ofrecer solamente la evaluación declarativa con la finalidad de continuar manteniendo su independencia respecto a las empresas, al no ser éstas sus clientes; con ello mantenían su compromiso con el inversor socialmente responsable, que era su auténtico cliente.

El problema que presentan las agencias que ofrecen los dos tipos de evaluación reside en que pueden llegar a perder su independencia, ya que harían auditoría (evaluación declarativa) y al mismo tiempo consultoría (evaluación solicitada). Tendrían que analizar a determinadas empresas para ofrecer la información a los inversores institucionales o a los gestores de fondos, y podría darse el caso de que estas empresas solicitaran ser analizadas para mejorar su RSC. Para solucionar este problema, hay agencias que, aun ofreciendo ambas evaluaciones, nunca las llevan a cabo en la misma empresa, ni simultánea ni consecutivamente: unas empresas son analizadas mediante evaluación declarativa, y otras mediante evaluación solicitada. Con ello, estas agencias no pierden su independencia. Con la evaluación declarativa estudian empresas que cotizan en bolsa, y con la evaluación solicitada ayudan a empresas que no cotizan en bolsa a mejorar en su responsabilidad social corporativa, normalmente pequeñas o medianas empresas.

Una de las diferencias a destacar entre las agencias norteamericanas y las europeas es la estructura del mercado. Las agencias de evaluación empezaron mucho antes en Estados Unidos que en Europa, y a lo largo de esa historia se fueron especializando en campos concretos de la ISR. De esta forma, cada agencia de evaluación de la RSC norteamericana se ha especializado en un perfil de cliente. Entonces, el 
mercado de las agencias de la RSC en Estados Unidos no es tan competitivo como el europeo, ya que en EEUU cada agencia tiene un sector de mercado. Pasemos ahora a analizar algunas agencias americanas.

En Estados Unidos hay cinco agencias de evaluación de la RSC 17: Calvert, Innovest, KLD, Domini Social Investment y Trillium. Podemos clasificar estas cinco agencias por sus productos:

1/ Innovest Strategic Value Advisors ofrece las evaluaciones declarativa y solicitada;

2/ la agencia KLD ofrece solamente la evaluación declarativa;

3/ y las agencias Domini Social Investments, Calvert y Trillium ofrecen fondos de inversión con criterios de RSC.

A continuación vamos a explicar las características de los tres grupos de agencias americanas.

\section{I. Innovest Strategic Value Advisors}

La agencia de evaluación de la RSC Innovest Strategic Value Advisor ${ }^{18}$ fue fundada por el Dr. Matthew Ciernan en 1995. Actualmente tiene sede en Nueva York, Toronto, Londres, París, San Francisco, Sydney y Tokyo. La agencia se define como una corporación de investigación en la rama de inversión, especializada en el análisis de indicadores de riesgo y valor accionariales, en el que incluyen el estudio del rendimiento de compañías en aspectos medioambientales, sociales y de gobernabilidad estratégica. Innovest hace evaluación solicitada y evaluación declarativa.

Todas las sedes de Innovest comparten la misma base de datos y utilizan la misma metodología para analizar empresas. Cada sede está organizada en distintos equipos de trabajo: cada equipo se especializa en un sector, $y$ analiza todas las empresas que pertenecen a dicho sector y que al mismo tiempo cotizan en bolsa. Por ejemplo, la sede de Nueva York tiene un equipo de trabajo especializado en banca. Este equipo está formado por cuatro personas, que analizan todos los ban-

\footnotetext{
${ }^{17}$ No analizamos aquí a Eiris, agencia con sede en la ciudad norteamericana de Boston, por ser una agencia propiamente europea.

18 INNOVEST (2008).
} 
cos. Las fichas que van a crear de cada banco van a ser incorporadas a su base de datos y por tanto podrán ser consultadas desde cualquier sede de Innovest. Así pues, cada sede se limita a evaluar las empresas de un sector determinado.

El estudio de cada compañía en particular es generalmente precedido de un análisis exhaustivo del sector industrial al que pertenece. Una vez evaluadas y puntuadas todas las empresas de un sector, Innovest procede a la atribución de la nota, que se escalona sobre 7 niveles de AAA a CCC. ${ }^{19}$

El análisis de Innovest se basa en los siguientes cuatro pilares:

a) Capital de los "stakeholders". Aquí se estudia la relación que tiene la empresa con la comunidad local, con los poderes públicos, con las ONGs, con los consumidores, con los socios aliados y con los mercados emergentes.

b) Gobernabilidad estratégica. Se analiza la política y la estrategia del desarrollo sostenible, la agilidad y la adaptación de la empresa, los indicadores de rendimiento, los aspectos de gobernabilidad tradicional y las prácticas internacionales.

c) Capital humano. Se estudia la gestión individual de los recursos humanos, las relaciones laborales, la salud y la seguridad, las estrategias de contratación, la motivación de los empleados, la capacidad de innovación y desarrollo y la difusión del conocimiento.

d) Medioambiente. Se analizan los sistemas de administración de riesgos medioambientales, la supervisión a cargo del consejo de administración y ejecutivo, la transparencia, la salud y seguridad y el desarrollo de nuevos productos.

\subsection{Calvert}

La agencia de evaluación Calvert, ${ }^{20}$ con sede en Bethesda, cerca de Washington DC, se fundó en 1976. Calvert ofrece fondos mutuales: concretamente ofrece 41

\footnotetext{
19 Standar \& Poor's define las letras de las calificaciones de las empresas como: "AAA: capacidad extremadamente fuerte de honrar los compromisos financieros. Es la calificación más alta. (...) CCC: Actualmente vulnerable y dependiente de condiciones favorables de negocios, financieras y económicas para poder cumplir con sus compromisos financieros". Cfr. StANDARD AND POOR's (2008).

${ }^{20}$ CALVERT (2008). 
fondos, de los cuales 22 son de ISR. La metodología que utiliza Calvert para analizar la RSC se llama Double Diligence ${ }^{\circledR}$, y su proceso de evaluación es el siguiente:

a) El encargado de crear una cartera de valores hace una lista de empresas con buen funcionamiento financiero, en las que estaría interesado en invertir.

b) Esta lista pasa al departamento de analistas de investigación de la inversión. Este departamento analiza la empresa según los siguientes siete criterios:

1. Gobernabilidad y ética. Calvert supervisa numerosos aspectos del gobierno corporativo, incluyendo políticas de empresa, independencia y diversidad de los dirigentes, remuneración ejecutiva, respuesta adecuada a las preocupaciones de los accionistas. Calvert se pone como objetivo no invertir en empresas que tengan estructuras de gobierno pobres o que dispongan de un expediente de actividades ilegales o cuestionables, como podrían ser el fraude, el soborno o la corrupción en general.

2. Medioambiente. Calvert evalúa la atención que la empresa presta al medioambiente, el grado de previsión de incidentes en este terreno, así como su capacidad de reacción ante ellos, y su conformidad con las regulaciones ambientales. Favorece a empresas que intenten atenuar su huella ambiental. Generalmente evita invertir en empresas implicadas en energía nuclear, a no ser que estas empresas hayan demostrado ser líderes en el desarrollo de la energía alternativa.

3. Lugar de trabajo. Este criterio comprende la salud y la seguridad del empleado, la diversidad de trabajadores y el tipo de relaciones laborales.

4. Seguridad del producto y su impacto. Calvert hace preselección negativa a empresas relacionadas con el tabaco, el juego, las armas, las bebidas alcohólicas o la experimentación abusiva con animales.

5. Derechos humanos. Se mide el grado de cumplimiento de los derechos humanos.

6. Derechos de los indígenas. Las empresas que operen directamente en tierra de indígenas, y las empresas cuyas operaciones afecten indirectamente a estas tierras, deberán apoyar el desarrollo económico del territorio.

7. Comunidad. Calvert evalúa positivamente a las empresas que han construido relaciones sólidas con la comunidad local. 
En cada criterio y en cada sector, hay un mínimo que la empresa evaluada por Calvert debe cumplir si quiere que Calvert invierta en ella.

c) Una vez finalizada la evaluación de la empresa en cuestión, la agencia entrega el correspondiente informe al encargado de crear una cartera de valores. En caso de que la empresa haya pasado satisfactoriamente la evaluación, el encargado de crear una cartera de valores decidirá si incluye o no a la empresa analizada en esta cartera. En cambio, en caso de que la empresa no haya aprobado la evaluación, esta empresa no podrá constar en ninguna cartera de valores de ISR de Calvert.

\subsection{KLD Research \& Analytics, Inc.}

KLD Research \& Analytics, Inc. ${ }^{21}$ con sede en Boston, fue fundada en 1988 por Peter D. Kinder y Amy L. Domini. Esta agencia se presenta a sí misma como la principal autoridad en la investigación social y en la elaboración de índices para inversores institucionales. KLD pertenece a Siri Company, un importante grupo de agencias de evaluación de la RSC22.

La misión de KLD es la siguiente ${ }^{23}$ :

- Proporcionar investigación a nivel mundial acerca de la RSC, y presentar un índice de productos que integre aspectos ambientales, sociales y de gobierno de la empresa en el proceso de inversión.

- Ofrecer productos y servicios que definan estándares de responsabilidad social de las empresas de forma que permitan a inversores, directores y fiduciarios influir en la conducta corporativa a través de sus decisiones de inversión y a través de la titularidad de las acciones.

- Lograr, a través de esta influencia, una mayor responsabilidad empresarial y, en definitiva, contribuir a crear una sociedad más justa y sostenible.

${ }^{21} \mathrm{KLD}$ (2008).

22 Los accionistas de Siri Company son las siguientes agencias de evaluación de la RSC: seis europeas (Centro Info, Scoris, Siri, Dutch Sustainability Research BV, GES Investment Services y Analistas Internacionales en Sostenibilidad), una canadiense (Jantzi Research Inc), una australiana (SIRIS), una israelí (Kayema Investment Research and Analysis) y una norteamericana (KLD).

${ }^{23} \mathrm{KLD}(2008)$. 
El inversor institucional o gestor de fondos europeo es distinto del estadounidense. En KLD esta diferencia se hace patente con sus dos métodos diferenciados de análisis de empresas: 1/ la metodología de Siri Company 2/ la metodología Socrates. KLD sólo analiza a empresas de Estados Unidos, y cuando analiza según la metodología de Siri Company, los informes sobre las empresas evaluadas son incluidos en la base de datos de Siri Company, y pueden ser consultados por cualquier accionista de Siri Company. Tengamos en cuenta que el $67 \%$ de los accionistas de Siri Company son europeos; la metodología utilizada en los análisis de empresas lleva el sello europeo de manera nítida.

KLD ha creado su propia metodología, bautizada con el nombre de Socrates. En la base de datos de Socrates el cliente de KLD puede consultar todas las empresas que pertenezcan a los índices bursátiles Rusell $3000^{\circledR}$ y S\&P $500^{\circledR}$.

Para analizar las empresas, KLD utiliza dos tipos de criterios, a saber:

1. Criterio RSC. Consiste en evaluar el impacto de la empresa en los distintos "stakeholders". Este análisis se apoya en los tres pilares siguientes: el pilar medioambiental, el pilar social y el pilar del gobierno de la empresa. Cada pilar será desglosado en distintos apartados, que a su vez serán atravesados por dos tipos de criterios: "strength" (fuerza) y "concern" (preocupación). En el Cuadro 8 "Criterios RSC de KLD" podemos ver los tres pilares mencionados, desglosados en algunos de los apartados que KLD utiliza en su análisis.

\section{CUADRO 8. Criterio RSC de KLD}

\begin{tabular}{|l|l|l|}
\hline Pilar medioambiental & \multicolumn{1}{|c|}{ Pilar social } & $\begin{array}{c}\text { Pilar de gobierno de la } \\
\text { empresa }\end{array}$ \\
\hline $\begin{array}{l}\text { Cambio climático } \\
\text { Productos y servicios }\end{array}$ & $\begin{array}{l}\text { Comunidad } \\
\text { Diversidad } \\
\text { Operaciones y } \\
\text { Relación con los } \\
\text { trabajiadración } \\
\text { Derechos humanos } \\
\text { Producto }\end{array}$ & $\begin{array}{l}\text { Informes públicos } \\
\text { realizados } \\
\text { Estructura de la } \\
\text { empresa } \\
\text { Transparencia }\end{array}$ \\
\hline
\end{tabular}

Fuente: documento interno de KLD proporcionado por la propia empresa en la entrevista del 15 de octubre de 2007. 
2. Criterio "Controversial Business Involvement" ("implicación en negocios polémicos"). Este criterio mide el nivel de implicación de la empresa en industrias involucradas en negocios polémicos, como podrían ser la industria del juego o del tabaco. KLD no invertirá en empresas relacionadas de un modo $u$ otro con los siguientes negocios: aborto, pornografía, alcohol, juego, tabaco, anticonceptivos, armas, ejército, energía nuclear con fines bélicos.

\section{A modo de conclusión: comparación sintética de las agencias estadounidenses con las agencias europeas}

Estados Unidos fue el país precursor de la ISR, y en la actualidad continúa siendo el país que más la promueve. Resulta impactante comparar la inversión tradicional realizada en Europa con la de Estados Unidos, ya que la americana supera en mucho a la europea. Este dato pone de relieve las notables diferencias culturales con respecto a la inversión que hay en Europa y en Estados Unidos: hay mucha más cultura de inversión en Estados Unidos que en Europa, por lo que no debe sorprender que la ISR sea muy superior en Estados Unidos que en Europa. Es necesario especificar que en Estados Unidos la ISR es superior a Europa tanto en valor absoluto como en porcentaje en relación a la inversión tradicional.

Además, el concepto de ISR es un poco distinto en Europa y en Estados Unidos. Tal y como hemos visto en el apartado 2, en Estados Unidos la ISR parte de la preselección de la cartera (sobre todo en filtro de selección negativo), las inversiones en comunidad y el activismo de los accionistas, mientras que en Europa parte de la preselección de la cartera (sobre todo en filtro de selección positivo), del compromiso (diálogo con las empresas), del activismo accionarial y del ejercicio del derecho de voto. En Europa, la inversión comunitaria no se considera ISR; en Estados Unidos, el activismo accionarial engloba el ejercicio del derecho de voto.

Actualmente, en Europa hay 23 agencias de evaluación de la RSC, mientras que en Estados Unidos sólo hay 5. Naturalmente, es complicado comparar Europa con Estados Unidos, ya que los Estados Unidos son un solo país, con un idioma común, con una notable unidad cultural interna, mientras que la Unión Europea está formada por muchos países, con idiomas muy distintos y con una notable diversidad cultural. No obstante, es útil conocer la realidad cuantitativa de las agencias de evaluación de la RSC en uno y otro sitio. Parece que la balanza esté claramente a favor de Europa si la comparamos en conjunto con Estados Unidos (23 contra 5). No obstante, si vamos país por país, resulta que en Europa podemos encontrar 
sólo una agencia de evaluación por país (hay países que no tienen ninguna y otros que tienen dos), mientras que en Estados Unidos, como hemos dicho, encontramos cinco. En esta comparativa, país por país, son los Estados Unidos los que salen claramente aventajados.

Hay tres agencias de evaluación americanas, Trillium, Calvert y Social Domini Investments, que funcionan de manera especial. Las incluimos en las agencias de evaluación porque analizan empresas de manera declarativa para ver su grado de responsabilidad social, pero de hecho ellas no hacen estos análisis para venderlos luego, sino sólo para uso interno de la agencia. Estas tres agencias son gestores de fondos, y hacen estos análisis para crear sus propias carteras de valores y ponerlas a disposición de sus clientes. En Europa no existe ni una sola agencia de estas características. Los clientes de estas agencias, al ser inversores, pasan a ser accionistas de las empresas y hacen activismo de los accionistas ("shareholder advocacy"), que consiste, como ya hemos dicho anteriormente, en el hecho que el inversor, al ser accionista de la empresa analizada, intente influir en el comportamiento de la empresa en cuanto a su responsabilidad social se refiere. Normalmente, estos inversores piden a la agencia de evaluación que sea ella quien contacte directamente con las empresas a invertir para influir en su comportamiento.

Las metodologías utilizadas para analizar la RSC de las empresas son similares en todas las agencias de evaluación, ya que todas quieren estudiar la repercusión que tiene en los stakeholders el comportamiento de una empresa. No obstante, podemos percibir diferentes tendencias en el modelo norteamericano con respecto al europeo. En Europa, el valor que se da al respeto al medioambiente es mucho más elevado que en Estados Unidos. Por el contrario, en Estados Unidos tiene un peso mayor el trato de igualdad dado a las minorías sociales en los lugares de trabajo. Por lo tanto, resulta importante recalcar que hay elementos comunes en las metodologías utilizadas para analizar la RSC en todas las agencias, aunque también es cierto que las distintas matrices culturales de cada país configuran distintos modelos de evaluación.

La primera agencia de evaluación de la RSC nació en 1976, en EEUU. Este país continúa siendo uno de los países que más impulsa la RSC. En cambio, en Europa, la primera agencia nació en 1983, en Londres, y en España no encontramos la primera agencia de RSC hasta el 2005. Por lo tanto se hace muy complicado considerar las agencias europeas como un todo, ya que hay tendencias distintas según los países. Lo que resulta claro es que EEUU es pionero y ha creado distintos tipos de agencias (Trillium, Calvert y Social Domini), que en Europa aún no existen. 


\section{Bibliografía y páginas web}

Albareda, L. - Balaguer, M.R. - Arenas D. (Coord.) (2007), Observatorio de la Inversión Socialmente Responsable en España 2006, Barcelona, IPES-ESADE.

Alberola, E. - Giamporcaro-SAUniÈre, S. (2006), "Les agences d'analyse et de notation extra-financière: quels services pour quels investisseurs?", Revue d'Économie Financière, n. 85, París, 171-189.

AlsINA, O. (Coord) (2002), La banca ética, mucho más que dinero, Barcelona, Icaria.

Avanzi SRI Research - Vigeo Italia (2007), "Green, social and ethical funds in Europe. 2007 Review", document on line, <http://www.vigeo.com>, consulta: 16 noviembre 2008.

AvaNZI SRI ReSEARCH (2005), "Green, social and ethical funds in Europe. 2005 Review", document on line, <http://www.avanzi-sri.org>, consulta: 30 octubre 2008.

AvanzI SRI ReSEARCH (2006), "Green, social and ethical funds in Europe. 2006 Review", document on line, <http://www.avanzi-sri.org>, consulta: 13 noviembre 2008.

Avanzi SRI Research, Vigeo (2007), "Green, social and ethical funds in Europe. 2007 Review", document on line, <http://www.eurosif.org/content/download/909/5094/version/1/file/Green+social+and+ethical+funds+in+Europe_2 007+Review.pdf>, consulta: 27 abril 2009

BalAguer, M.R. (2006), "La inversión socialmente responsable en España: tres ensayos", Tesis Doctoral, Castellón, Universitat Jaume I.

BASCONES, P. (2006), "Los índices bursátiles de sostenibilidad y la inversión socialmente responsable", Ecosostenible, n. 21, Barcelona, 9-19.

CALVERT (2008): <www.calvert.com>, período de consulta: junio 2007 - noviembre 2008.

Camprodon, M. - Sols, J. - Florensa, A. (2006), "Las agencias de evaluación de la Responsabilidad Social Corporativa: estudio de un caso", Revista de Fomento Social, n. 244, vol. 61, Córdoba, 393-422. 
Camprodon, M. - Sols, J. - Florensa, A. (2007), "La necesidad de las agencias de evaluación de la RSC", Economía Social, n. 41, Madrid, 10-13.

DéJEAN, F. (2005), L'investissement socialement responsable. Étude du cas français, París, Vuibert.

DuPRÉ, D. - GIRERD-POtIN, I. (2002), "L'essor d'une consomation éthique", en: Dupré, D., Éthique et Capitalisme, París, Economica, 85-119.

EIRIS (2007): <www.eiris.org>, período de consulta: junio 2007.

EUROSIF (2006), "European SRI Study", documento on line, <http://www.eurosif. org>, consulta: 14 noviembre 2007.

First Affirmative Financial Network, documento on line <http://www.firstaffirmative. com/news/sriArticle.jsp>, consulta: 27 abril 2009.

Henderson, H. - Lickerman, J. - Flynn, P. (2000), Calvert-Henderson. Quality of life indicators, Bethesda, Calvert Group.

INNOVEST (2008), <www.innovestgroup.com>, período de consulta: junio 2007 noviembre 2008.

Kawamura, M. (2002), "How Socially Responsible Investment (SRI) Could Redefine Corporate Excellence in the $21^{\text {st }}$ Century", NLI Research Institute, n. 160, Tokio, $12-23$.

KLD (2008), <www.kld.com>, período de consulta: junio 2007 - noviembre 2008.

LOUCHE, C. - LYDENBERGS. (2006), "Investissement socialement responsable: différences entre Europe et États-Unis", Revue d'Économie Financière, n. 85, París, 81-105.

NOVETHIC (2007), <http://www.novethic.fr>, consulta: 2 diciembre 2007.

ORSE - ADEME (2006), "Guide des organismes d'analyse sociétale et environnementale", documento on line, <www.orse.org>, consulta: 18 junio 2008.

Sánchez, P. - Rodríguez, M.A. - Ricart, J.E. - Capdeville, S. - Gal, E. (2004), "La Inversión Socialmente Responsable: evolución, tendencias e implicaciones para 
la dirección de empresas", Documento de Investigación, n. 464, IESE Business School.

SOcial InVESTMENt Forum (2006), "2005 Report on Socially Responsible Investing Trends in the United States, 10 years review", documento on line, <http://www. socialinvest.org>, consulta: 24 noviembre 2008.

STANDARD AND POOR's (2008): <http://www2.standardandpoors.com>, período de consulta: agosto 2008. 